\title{
The consistency approach for the quality control of vaccines
}

\author{
Coenraad Hendriksen ${ }^{a, b}$, Juan L. Arciniega ${ }^{c}$, Lukas Bruckner ${ }^{\text {d }}$, Michel Chevalier ${ }^{\text {e, }}$ \\ Emmanuelle Coppens ${ }^{\mathrm{e}}$, Johan Descamps ${ }^{\mathrm{f}}$, Michel Duchêne ${ }^{\mathrm{f}}$, David Michael Dusek ${ }^{\mathrm{g}}$, \\ Marlies Halder ${ }^{\mathrm{h}}, *$, Hans Kreeftenberg ${ }^{\mathrm{a}}$, Alexandrine Maes ${ }^{\mathrm{i}}$, Keith Redhead ${ }^{\mathrm{j}}$, \\ Satish D. Ravetkar ${ }^{k}$, Jean-Marc Spieser ${ }^{1}$, Hanny Swam ${ }^{\mathrm{m}}$ \\ ${ }^{a}$ Netherlands Vaccine Institute, A. van Leeuwenhoeklaan 11, 3720 AL Bilthoven, The Netherlands \\ ${ }^{\mathrm{b}}$ Netherlands Centre Alternatives to Animal Use, Utrecht University, 3508 TB Utrecht, The Netherlands \\ ${ }^{\mathrm{c}}$ Center for Biologics Evaluation and Research, Food and Drug Administration, 1401 Rockville Pike, Rockville, MD 20852-1448, USA \\ d Institute of Virology and Immunoprophylaxis, CH-3147 Mittelhaeusern, Switzerland \\ e Sanofi Pasteur, 1541 avenue M. Mérieux, 69280 Marcy l'Etoile, France \\ ${ }^{\mathrm{f}}$ GlaxoSmithKline Biologicals, Rue de l' Institut 89, B-1330 Rixensart, Belgium \\ ${ }^{\mathrm{g}}$ U.S. Department of Agriculture, Animal and Plant Health Inspection Service, Center for Veterinary Biologics, Policy, Evaluation and Licensing, \\ 510 South 17th Street, Ames, IA 50010, USA \\ ${ }^{\mathrm{h}}$ European Centre for the Validation of Alternative Methods, Institute for Health and Consumer Protection, Joint Research Centre, European Commission, \\ TP 580, Via Fermi 1, 21020 Ispra, Varese, Italy

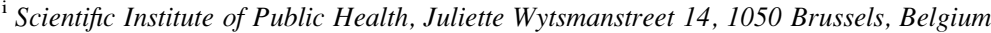 \\ ${ }^{\mathrm{j}}$ Intervet UK Ltd, Milton Keynes MK7 7AJ, United Kingdom \\ ${ }^{\mathrm{k}}$ Serum Institute of India Ltd, 212/2 Hadapsar, Pune 411028, India \\ ${ }^{1}$ European Department for the Quality of Medicines, Council of Europe, 7 allée Kastner, CS 30026, 67081 Strasbourg, France \\ m Intervet International BV, PO Box 31, 5830 AA Boxmeer, The Netherlands
}

Received 29 March 2007; revised 2 May 2007; accepted 5 May 2007

\begin{abstract}
Current lot release testing of conventional vaccines emphasizes quality control of the final product and is characterized by its extensive use of laboratory animals. This report, which is based on the outcome of an ECVAM (European Centre for Validation of Alternative Methods, Institute for Health and Consumer Protection, European Commission Joint Research Centre, Ispra, Italy) workshop, discusses the concept of consistency testing as an alternative approach for lot release testing. The consistency approach for the routine release of vaccines is based upon the principle that the quality of vaccines is a consequence of a quality system and of consistent production of lots with similar characteristics to those lots that have been shown to be safe and effective in humans or the target species.

The report indicates why and under which circumstances this approach can be applied, the role of the different stakeholders, and the need for international harmonization. It also gives recommendations for its implementation.
\end{abstract}

(C) 2007 The International Association for Biologicals. Published by Elsevier Ltd. All rights reserved.

Keywords: Vaccines; Quality control; Consistency; Release testing; In vitro

\footnotetext{
* Corresponding author. Tel.: +39 0332785550; fax: +390332785336.

E-mail address: marlies.halder@jrc.it (M. Halder).
}

\section{Introduction}

The European Centre for Validation of Alternative Methods (ECVAM, Institute for Health and Consumer, Protection, European Commission Joint Research Centre, Ispra, Italy) organised a workshop on the consistency approach and its potential 
to reduce the number of animal tests used in the quality control of vaccines. The workshop took place on 17-19 May 2006 in Ispra, Italy.

A key element of current vaccine manufacturing practice is the consistent production of vaccine lots with similar characteristics to those lots shown to be safe and effective in the target species. The consistency principle is implicit in the licensing/registration process and was already identified early in the 20th century [1]. Adherence to this principle has resulted in the use of reference preparations, test guidelines and release criteria for those characteristics relevant for safety and efficacy. At the manufacturing level, quality systems ensuring consistency of production, such as current Good Manufacturing Practices (cGMP, which include parametermonitoring) and quality assurance (QA) principles, are now common. The ultimate goal of the quality control and regulation of prophylactic and therapeutics agents is to ensure that only safe and effective products appear on the market.

Characterisation of vaccines is more difficult than characterisation of pharmaceuticals, due to the complex molecular structure of the antigens they contain, their production processes, and their interaction with agents used during manufacturing and/or present in the final lot, such as preservatives and adjuvants. Consequently, in vivo quality control of vaccines has been more extensive, with emphasis on testing of the final product with animal assays purportedly predictive of product behavior in target populations. As a result, lot release testing of vaccines requires substantial numbers of laboratory animals of various species. It is estimated that worldwide use exceeds 5 million animals per year.

For more recently developed vaccines (e.g. polysaccharide conjugate vaccines, such as haemophilus $b$, pneumococcus and meningococcus vaccines), a better handle on consistency in production through in-process testing has allowed simplification of release protocols [2], in particular related to the use of in vitro tests, whilst maintaining the capacity to demonstrate equivalency with the clinical lots. We believe that, for various reasons, the concept can be extended to vaccines that have already been on the market for quite a long time (e.g. toxoid vaccines, polio vaccines, Leptospira vaccines); however, some aspects have still to be examined [3].

The workshop discussed the following topics: what is the consistency approach, what are the elements of this approach, what are the quality standards for implementation, what are the pitfalls, who are the stakeholders and what are their respective roles and finally, what is the roadmap to successful implementation? The outcome of these discussions and the recommendations given by the workshop participants are summarised in this report.

\section{Consistency approach: definition and reasons for use}

Consistency is an old concept that aims to ensure the uninterrupted release of safe and efficacious products. The following performance description of consistency can be given when used as a new approach to routine lot ( $=$ batch) release testing of the traditional vaccines: The consistency approach implies the use of a set of parameters to constitute a product profile (e.g. antigen content, antigen integrity, etc.) that can replace current release tests. The product profile is established to the satisfaction of the regulators at the time of licensing, and is monitored throughout production under a strict quality system. The product profile ensures that each batch or lot released is similar to a manufacturer-specific vaccine of proven clinical efficacy and safety, with respect to all characteristics agreed upon at licensing between manufacturer and regulator. This approach may lead to a reduction in animal use, since a narrow set of animal bioassays per final lot, with potentially limited power to predict vaccine behavior in the target populations, may be substituted by a battery of meaningful tests with enhanced capacity to measure equivalence with lots of proven safety and efficacy [4]. The consistency approach makes use of progress that has been made in bioanalytical and other assay methods $[5,6]$.

Quality systems applied during vaccine production, as well as accompanying analytical tools, have experienced considerable improvement in their ability to monitor the consistency of critical characteristics of vaccines from batch to batch (cGMP, QA, etc.) [7]. Therefore, the workshop concluded that the consistency approach could now be within the realm of routine lot release testing. Additional reasons to support this paradigm shift are associated with aspects or flaws of the animal model used:

- Relevance: We now better understand the limitations of laboratory animal models to measure vaccine characteristics relevant for predicting their safety and efficacy in humans and target animals (in the case of veterinary vaccines). In fact, several of the models in use have never been properly validated as regards their predictive value for clinical efficacy [8].

- Ethics and costs: The use of laboratory animals poses ethical concerns, is expensive, time consuming and requires skilled staff and sophisticated experimental facilities.

- Reference preparation: Some of the currently used animal models that include the use of an international reference preparation do not meet the fundamental criteria defined by Jerne and Maaløe [9] for bioassays that test and reference vaccine should behave as unknown dilutions of each other.

It is believed that the extension of the consistency approach to conventional products will (a) introduce a more stringent system to manage the quality of the vaccine lots produced and (b) lead ultimately to a substantial reduction in animal usage. This approach aims to identify the critical indicators of a safe and effective product and to monitor these indicators during production, thus reducing the need for extensive final lot testing, often associated with the use of animals. Consistency builds on elements of information obtained during product development (including clinical testing) and routine production using suitable analytical tools and the continuous evaluation of data generated over time (trend analysis). 


\section{Quality elements to be considered for implementation of the consistency approach}

In order to limit or eliminate in vivo testing through the building of a consistency approach for lot release purposes, the following elements should be evaluated.

\subsection{Clinical criteria}

The profile of all lots for commercial release should be consistent with the profile of lots shown to be efficacious and safe in clinical studies performed under current Good Clinical Practices (cGCP). This approach is applicable to newly developed products. For older products for which clinical data were not established according to cGCP, one could refer to historical data and post-marketing surveillance information, which must have been suitably designed for the purpose.

\subsection{Manufacturing criteria}

Vaccine lots should have been demonstrably produced in a consistent way according to the approved registration file and following cGMP. This involves all production steps, appropriate process validation, environmental monitoring, personnel qualifications and QA supervision, including internal audits and inspections performed by Regulatory Authorities.

\subsection{Testing criteria}

Only scientifically meaningful, non-redundant tests that measure characteristics associated to safety and efficacy, as well as additional tests that address consistency of the production process, should be included in the generation of the product profile. The tests must be properly validated and performed in the context of a QA system. This product profile should be discussed as early as possible with the regulatory bodies. It should be noted that although release specifications should be included in the product profile, tighter limits for the same parameters might be used to establish and monitor consistency, as it is described for example in the European Pharmacopoeia (Ph.Eur.) monograph for human vaccines [10]; while release specifications are mainly based on the behavior of the clinical consistency lots in the tests, consistency limits are based on trending data.

For more complex vaccines, containing conventional components and/or certain types of adjuvant, it may be necessary to perform limited immunogenicity studies in a small number of animals as part of the final product testing. Also, if manufacturing changes occur, the need for in vivo testing, at least for a limited period through which consistency is being re-demonstrated, should be re-assessed.

\subsection{Application criteria}

The most obvious application of the consistency approach is in the routine release of product. However, this approach could also be applicable to stability testing under certain circumstances. For example, the approach could be applied during stability studies to confirm consistency with the product profile. For a specified number of lots this might be complemented by re-assessment of stability by in vivo testing. It should nevertheless be clear that if such an approach were to become current practice the system should be rethought for certain kinds of products.

\section{Quality standards in the consistency approach}

Testing is only one component of an operational quality system, which includes careful validation and maintenance of the manufacturing process that produced lots of vaccine shown to be clinically safe and effective. Nowadays it is a well-established philosophy that the quality of a vaccine is not ensured by testing alone, but rather built through the strict application of the quality assurance system. The consistency approach reinforces this vision and extends it to other activities in the vaccine life-cycle, such as the field history of the vaccine, established before licensing by clinical studies of more than one lot of product on trial, and after licensing, by programs of pharmacovigilance. The importance of complete and accurate written procedures and the existence of a system that evaluates the effect of changes in the production process cannot be over emphasized.

As mentioned above, although release specifications are useful tools to establish consistency of manufacturing, other in-process characteristics and parameters measured on the intermediates and the final product may also contribute to the assessment of consistency. Manufacturers should have established, as part of a quality assurance system, procedures and set control limits (e.g. alert limits, action limits) for controlling the performance of any meaningful parameter over time. Release specifications should not be confused with quality control limits. Release specifications are defined during product development and tend to be less conservative than some quality control limits, although both are based on the accumulated experience of both the manufacturers and the regulators. Sometimes both types of parameters are set on a provisional basis at the time of licensing and can only be fully defined after a sufficient number (decided on a case-by-case basis) of lots are manufactured. For the same parameter, the buffer zone between quality control limits and release specifications allow the manufacturer to investigate deviation from control limits and correct their root causes without sacrificing numerous batches. Additionally, the existence of quality control limits, in addition to specifications, allows the manufacturer to improve consistency over time, without the need for a formal change in specifications that could entail the performance of additional clinical or animal studies.

Extended acceptance of the consistency approach will require a profound review of the current system of international standardization [11]. A fundamental first step in international harmonization is the recognition of the validity of the proposed approach. Once the approach has been endorsed internationally, publication of guidelines explaining its operative details is of utmost importance. 
One of the most misunderstood parts of the consistency approach is the role of physical standards in potency testing. Because the consistency approach intends to establish a link between the clinical lots and subsequent batches of vaccine, it is often assumed that there is a need for a stable clinical lot that could be used as standard or control. In reality, what is assessed in the consistency approach is the similarity of the potency or activity of every batch manufactured after licensing to that of one or more lots shown to be safe and effective. For this purpose, if the manufacturer and the regulatory authority so decide, limits of acceptance can be calculated relative to a standard vaccine of known behavior. This batch of vaccine may or may not be a clinical lot, and can be replaced whenever it becomes necessary, provided that the activity of the new reference is adjusted with respect to that of the old reference. This is similar to the way International Standards are currently used. However, it should be noted that results obtained for one type of vaccine may not be comparable with results obtained for a different type of vaccine, if what the potency test is measuring is not a surrogate of efficacy in the target population; therefore, homologous standard vaccines for each type of product may be required. A multinational organization could maintain and distribute these reference vaccines.

\section{Potential and perceived pitfalls of the consistency approach}

Because even modern vaccines are products of a complex nature, the use of non-animal alternatives for the laboratory prediction of their efficacy in the field is considered sometimes to be imperfect. Whilst the consistency of biochemical or immunological characteristics of individually purified antigens or separable antigenic components can be established with relative ease, the combination of antigens and the addition of excipients could hinder the meaningful use of in vitro testing to assess antigen quality in the final product [12], although new developments might allow progress in this area [13,14]. This limitation is invoked to justify the use of a full-blown relative potency test in animals for the batch release of at least some vaccines. However, the impact of the potential shortcomings of in vitro testing, or limited animal testing, on product characterisation may be lessened in the consistency approach by the use of all relevant information available throughout the manufacturing process from raw materials through the final formulation step. Moreover, although in some instances the value of animal testing cannot be denied, in others its power to predict safety and/or efficacy in the target population is limited. If the predictive power of an animal test is limited, the consistency approach favors the selection of an alternative, since in this approach any test, including refined animal tests for potency applied to the final product, is only part of a battery of assays scientifically designed to provide the greatest assurance that the vaccine will be effective in the target species. Care should be exercised when deciding on the acceptable outcome to be measured in the tests composing the product profile. These tests must be relevant and indicative of the quality and quantity of the product. Simplification and/or disregard of the scientific findings in an effort to reduce or eliminate the use of animals in potency testing must be avoided in order to maintain the credibility of the consistency approach.

While routine batch release is generally amenable to the consistency approach, sometimes the use of fully developed animal-based potency tests may offer obvious advantages. For example, on those occasions when a test with good predictive power for efficacy is used during licensing or is applied to justify major changes in vaccine formulation or manufacturing processes of a licensed vaccine.

\section{Role of stakeholders and need for harmonization}

For many years the recommendations adopted by the World Health Organization (WHO) Expert Committee on Biological Standardization (ECBS) have been used as the basis for National Regulations for vaccines for human use around the world. On the other hand, while the Ph.Eur. elaborates product monographs mandatory only for countries that signed the convention of the Ph.Eur., WHO has often followed approaches closely resembling those included in Ph.Eur. documents, particularly when modern, better-characterized products are described. The participants of the workshop are well aware that method harmonization is more difficult at the world level, owing to different levels of technical development. However, they propose that Ph.Eur. takes the lead in encouraging $\mathrm{WHO}$, in their frequent formal and informal interactions, to unequivocally endorse the consistency approach for vaccine regulation, reinforcing also the sponsorship of the Three Rs as a guiding principle for test development.

Adopting the consistency approach will confer flexibility to the thought process through which a manufacturer selects an alternative for its inclusion in the product profile to be discussed with the regulator during licensing. If the status quo remains in place, the practice in some countries of adopting vaccine-testing strategies inconsistent with WHO recommended designs will continue and may even expand.

Participants believe that, on occasion, vaccine manufacturers and regulators in developing countries and those with emerging economies oppose the adoption of in vitro alternatives because of the misconception that animal testing is less expensive and easier to do than in vitro testing. However, the costs associated with breeding, housing and handling of animals in conditions that meet current standards are often overlooked. On the other hand, it was recognized that sometimes the purchase costs of reagents and equipment used for alternative tests, such as ELISA, could be prohibitive.

It was also acknowledged that some degree of consistency of manufacturing is currently included in the contract established between manufacturers of products for animal use and the corresponding regulatory authority. Although manufacturers of these types of products may not be bound by cGMP, which only apply in a strict sense to the manufacturing of vaccines for human use, analogous regulations are in place in some countries and the situation is still evolving. 
It was agreed that ICH and VICH-generated documents have played a significant role in harmonizing procedures and concepts among participants of these multilateral organizations. However, the impact of their decisions on the design of vaccine quality policy in less developed countries is reduced, because both organizations are perceived as catering to the interests of official members, which are all developed countries.

The group recognized that the WHO and Ph.Eur. practice of providing reference materials to which international units of activity have been assigned may have to be abandoned for certain products regulated through the consistency approach. However, there may still be a need to ensure the availability of some reagents (e.g. reference sera or monoclonal antibodies).

\section{Recommendations}

1. Harmonization of the consistency approach between Manufacturers and Regulatory Authorities (e.g. European Union Member States, WHO, USA) is required. This can be achieved by communication between all involved parties at an early stage and the establishment of international guidelines (WHO). There should be a mechanism in place for training the different parties to ensure a homogenous approach.

2. The consistency approach should be seen as a dynamic process that re-evaluates and/or adapts the product profile.

3 . The implementation process should consist of: (a) definition of the product profile on the basis of equivalence to clinically tested lots and in agreement with the Regulatory Authorities, (b) generation of data on a suitable number of lots, (c) introduction of a variation file, and (d) approval by the Regulatory Authorities. International recognition is a way to establish harmonization.

4. Analytical tools and performance standards should be developed that are able to demonstrate any deviation from consistency of production and any change in product profile agreed upon at licensing.

5. Sub-potent lots can be a useful tool during the development phase of analytical tests. These lots can be used for validation purposes. However, there is no need to use them in routine lot release testing.

6. There should be an incentive at both the level of the Manufacturer and the Regulatory Authorities to move towards this consistency approach. Lack of appropriate in vitro analytical tools for consistency testing should encourage a special program of research. In this context, the importance of the economic impact must not be neglected.

\section{References}

[1] Virus-Serum-Toxin Act. The Act of March 4, 1913, <http://www.aphis. usda.gov/vs/cvb/html/vsta.html>. 37 Stat. 832-833.

[2] Jones C, Currie F. Control of components of bacterial polysaccharide vaccines by physical methods. Biologicals 1991;19:41-7.

[3] Metz B, Brunel F, Chamberlin C, van der Gun J, Halder M, Jiskoot W, et al. The potential of physicochemical and immunochemical assays to replace animal tests in the quality control of toxoid vaccines. ATLA 35 , in press.

[4] Hendriksen CFM. Towards eliminating the use of animals for regulatory required vaccine quality control. ALTEX 2006;23:187-91.

[5] Metz B, Jiskoot W, Hennink WE, Crommelin DJ, Kersten GFM. Physicochemical and immunochemical techniques predict the quality of diphtheria toxoid vaccines. Vaccine 2003;12:156-67.

[6] Metz B. Structural characterisation of diphtheria toxoid. Thesis. PrintPartners Ipskamp B.V., Enschede, Netherlands; 2005. ISBN: 90-393-3941-4.

[7] Dellepiane N, Griffiths E, Milstien JB. New challenges in assuring vaccine quality. Bull World Health Organ 2000;78:155-62.

[8] Hendriksen CFM, van der Gun J. Animal models and alternatives in the quality control of vaccines: are in vitro methods or in vivo methods the scientific equivalent of the emperor's clothes. ATLA 1995;23:61-73.

[9] Jerne NK, Maaløe O. Standardization of diphtheria toxoid. Some theoretical and practical considerations. Bull World Health Organ 1949;2: 49-57.

[10] Council of Europe. Vaccines for human use, European pharmacopoeia. 5th ed. Strasbourg, France: Council of Europe; 2006. Suppl. 5.4, $3838-3840$.

[11] Milstien JB. Regulation of vaccines: strengthening the science base. J Public Health Policy 2004;25:173-89.

[12] Metz B, Hendriksen CFM, Jiskoot W, Kersten GFM. Reduction of animal use in human vaccine quality control: opportunities and problems. Vaccine 2002;20:2411-30.

[13] White JL, Helm SL. Characterization of aluminium-containing adjuvants. Dev Biol 2000;103:217-28.

[14] Wittayanukulluk A, Jiang D, Regnier FE, Hem SL. Effect of microenvironment $\mathrm{pH}$ of aluminium hydroxide adjuvant on the chemical stability of adsorbed antigen. Vaccine 2004;22:1172-6. 